hep-ph/0507xxx

July 2005

\title{
Non-Perturbative Instabilities as a Solution of the Cosmological Moduli Problem
}

\author{
Natalia Shuhmaher and Robert Brandenberger \\ Department of Physics, McGill University, Montréal, QC, H3A 2T8, CANADA
}

\begin{abstract}
It is widely accepted that moduli in the mass range $10 \mathrm{eV}-10^{4} \mathrm{GeV}$ which start to oscillate with an amplitude of the order of the Planck scale either jeopardize successful predictions of nucleosynthesis or overclose the Universe. It is shown that the moduli problem can be relaxed by making use of parametric resonance. A new non-perturbative decay channel for moduli oscillations is discussed. This channel becomes effective when the oscillating field results in a net negative mass term for the decay products. This scenario allows for the decay of the moduli much before nucleosynthesis and, therefore, leads to a complete solution of the cosmological moduli problem.
\end{abstract}

PACS numbers: 98.80.Cq.

\section{INTRODUCTION}

In many theories beyond the Standard Model of particle physics, in particular in supergravity and string theories, there are many scalar and fermionic fields with masses smaller or equal to the electroweak scale and gravitational strength couplings to ordinary matter. Such fields are called moduli fields and behave as non-relativistic matter at late time. Since they decay late because of their weak interactions, they lead to the so-called cosmological moduli problem $[1,2,3,4]$. Below, independently of their origin, fields having only Planck scale couplings and weak scale mass are denoted collectively as moduli fields.

In the case of moduli in supersymmetric theories, the mass $m_{\phi}$ of these fields $\phi$ is generated during supersymmetry breaking. A wide variety of these scenarios predict masses of the moduli in the dangerous range, $m_{\phi} \sim 10 \mathrm{eV}-10^{4} \mathrm{GeV}$. According to our ideas of early Universe cosmology, we expect moduli to be produced in great abundance in the early universe. In the context of Big Bang cosmology, both scalar and fermionic moduli particles will be part of the initial thermal bath of particles of the very early universe. Even assuming that the moduli particles are not part of the initial thermal bath (for example in the context of inflationary cosmology) it is hard to avoid the presence of excited moduli fields at late times. For example, in the case of scalar moduli, since the moduli are massless before supersymmetry breaking, there is no reason that the moduli field values before supersymmetry breaking coincide with the values which turn into the minima of the potential after supersymmetry breaking [5]. The offset will lead to moduli fields which oscillate about their potential minima. An offset of a scalar modulus can also be produced by quantum fluctuations in the early phases of inflation, as follows from the computation of the coincident point two point function of a low-mass scalar field during inflation $[6,7]$. The excessive production of moduli is predicted during the preheating stage of inflationary cosmology in a wide variety of models [8]. A further source of moduli particles is gravitational particle production between the end of inflation and the time of nucleosynthesis $[9,10]$.

Due to their weak interactions, the decay of the moduli fields is slow. Widely used estimates based on dimensional analysis for the perturbative decay rate $\Gamma$ give

$$
\Gamma \sim \frac{m_{\phi}^{3}}{M_{p}^{2}}
$$

The presence of excited moduli fields at late times is dangerous since the presence of the extra moduli field energy during the time of nucleosynthesis could spoil the success of the standard Big Bang nucleosynthesis scenario [11]. This danger is acute in particular for the heavier moduli fields. Both lighter moduli fields and heavier fields which do not decay before the time of equal matter and radiation threaten to overclose the Universe at that time (and thus also today if they do not decay between the time of equal matter and radiation and the present time).

To be more specific, it is the fact that 
the interactions of moduli fields come from non-renormalizable terms in the supersymmetry (SUSY) potential that leads to the moduli problem. For example, the following term in the Kähler potential

$$
\delta K=\frac{1}{M_{p}^{2}} \phi_{I}^{\dagger} \phi_{I} \phi^{\dagger} \phi
$$

results in a contribution to the square mass for $\phi$ of the form $\rho / M_{p}^{2}$ [12]. By $\phi$ we denote a canonically normalized modulus field (with bare mass $m_{\phi}$ ), by $M_{p}$ - the Planck mass, by $\phi_{I}$ - a field which dominates the energy density of the universe, the inflaton, and by $\rho$ - the energy density contained in the inflaton. Since $\rho=3 H^{2} M_{p}^{2}$, the effective mass $m_{\text {eff }}$ becomes

$$
m_{\text {eff }}^{2}=c H^{2}+m_{\phi}^{2},
$$

where $c$ is a constant. If $c \gg 1$, then inflation drives the moduli to the minima of their high temperature effective potential. However, typically the high temperature minima are offset from the zero temperature minima of the moduli potentials by a value which has Planck order of magnitude. If $c \ll 1$ then $H>m_{e f f}$, and then quantum fluctuations during inflation will also excite the field $\phi$ to a value of the order of the Planck mass. During reheating the energy density of the inflaton stops dominating the Universe and the effective mass of $\phi$ relaxes to $m_{\phi}$. After reheating, the Hubble constant decreases in the radiation dominated phase. Once $H \sim m_{\phi}$ the condition for slow rolling of $\phi\left(V^{\prime \prime}(\phi)<H^{2}\right)$ is no longer satisfied, and at that point the field $\phi$ starts to oscillate around its low temperature minimum which we take to be zero. The energy density of $\phi$ decreases like that of non-relativistic particles (i.e. proportional to $a(t)^{-3}$, where $a(t)$ is the cosmological scale factor), whereas the energy density of the radiation dominated universe falls as $a(t)^{-4}$. Thus, the modulus field $\phi$ may come to dominate the energy density of the universe, or at least contribute too much during the period of nucleosynthesis, unless the field decays early. The problem is that the modulus fields are coupled only gravitationally to themselves and other particles and thus have very small perturbative decay rates.

There has been some previous work to try to mitigate the cosmological moduli problem. En- tropy production at late times will dilute the moduli density. For example, weak scale inflation [13] or thermal inflation [14] could sufficiently dilute unwanted moduli (see e.g. [15] for a detailed study of the potential of thermal inflation to solve the moduli problem for masses in the above-mentioned dangerous range predicted in models with hidden sector and gauge-mediated supersymmetry breaking). For certain ranges of parameters of a gaugemediated supersymmetry breaking model, the oscillations of the modulus field itself might sufficiently dilute the string moduli density [16]. The decay of an unstable domain wall network [17] is another way to generate entropy and dilute the moduli density at late times. A common danger of these approaches is that the baryon density might also be diluted to an unacceptably low value. Another approach is to invoke effects which give the moduli fields a contribution to the square mass of the order of $H^{2}$ which would allow them to roll down their potential during inflation $[18,19]$ and prevent them from acquiring a large expectation value during inflation (see also [20]). However, this solution does not work if the low temperature minimum of the moduli potential does not coincide [5] with its high-temperature ground state (new symmetries which could force the two states to be the same were analyzed in [12]). A recent proposal to solve the moduli problem is moduli trapping at enhanced symmetry points $[21,22]$. In terms of the use of parametric resonance instabilities, our work has similarities with that of $[21,22]$. However, in contrast to these works, in our study the focus is on the traditional moduli problem as formulated in $[1,2,3,4]$.

In this paper, we propose a way to solve the cosmological problems of scalar moduli fields which requires no external mechanism for the dilution of moduli. Instead, it makes use of non-perturbative decay channels. Non-perturbative decays have been shown to completely change the scenario of reheating in inflationary cosmology. In particular, the decay of the inflaton field by a parametric resonance instability has been shown to be very important $[23,24,25,26]$. In certain models, a tachyonic instability renders the inflaton decay even more efficient [27].

We consider a toy model which potentially gives rise to a cosmological moduli problem. In the framework of this model we investigate non- 
perturbative decay channels (decays into particles whose lifetime is much shorter than that of the moduli fields). We study the decay of the oscillating modulus field via parametric resonance, and propose a new tachyonic decay channel. We quantify the conditions on the parameters of the model for which the decay channels are effective. Note that the decay channels work for an initial field amplitude up to the order of the Planck scale. No external mechanism for diluting the moduli density is required.

\section{THE MODEL}

If the moduli problem arises as a consequence of supersymmetry breaking, the moduli potential takes the form (see e.g. [13])

$$
\mathcal{V}(\Phi)=m_{3 / 2}^{2} M_{p}^{2} \mathcal{G}\left(|\Phi| / M_{p}\right),
$$

where $m_{3 / 2}$ is the gravitino mass and $\mathcal{G}$ is some function. We will assume that the modulus field couples to some matter field $\chi$ which we treat as a scalar field (following the analyses in the study of fermionic preheating [28] it could also be taken to be a fermionic field). Making use of (4), and of the fact that the dimension five and six operators are suppressed by $M_{p}$ and by $M_{p}^{2}$, respectively, we can construct a typical potential for the modulus $\phi$ and the matter scalar field $\chi$

$$
\begin{aligned}
V= & \frac{1}{2} m_{\phi}^{2} \phi^{2}+\frac{1}{2} m_{\chi}^{2} \chi^{2}+\frac{1}{2} \frac{m_{I}^{2}}{M_{p}} \phi \chi^{2} \\
& +\lambda_{1} \phi^{2} \chi^{2}+\lambda_{2} \chi^{4}+\lambda_{3} \phi^{4}
\end{aligned}
$$

where the coupling constants $\lambda_{1}, \lambda_{2}, \lambda_{3}$ are small enough such that for $|\phi|,|\chi|<M_{p}$ the low energy effective potential for $\phi$ takes form

$$
V_{l}=\frac{1}{2} m_{\phi}^{2} \phi^{2}+\frac{1}{2} m_{\chi}^{2} \chi^{2}+\frac{1}{2} \frac{m_{I}^{2}}{M_{p}} \phi \chi^{2} .
$$

Thus, the toy model we consider is described by the following Lagrangian:

$$
\begin{aligned}
L= & \partial^{\mu} \phi \partial_{\mu} \phi+\partial^{\mu} \chi \partial_{\mu} \chi \\
& -\frac{1}{2} m_{\phi}^{2} \phi^{2}-\frac{1}{2} m_{\chi}^{2} \chi^{2}-\frac{1}{2} \frac{m_{I}^{2}}{M_{p}} \phi \chi^{2} .
\end{aligned}
$$

In the above, the mass $m_{I}$ sets the scale of the interaction between $\phi$ and $\chi$. It is reasonable to assume that this scale is much less than $M_{p}$.

In analogy to the situation encountered in the study of the decay of the inflaton, if we want to study the decay of the modulus field, it is crucial to focus on the equation of motion for matter fields $\chi$ which the modulus field couples to. In the presence of the oscillating modulus field, this equation (in an expanding space-time) is

$$
\begin{aligned}
\ddot{\chi}_{k} & +3 H \dot{\chi}_{k}+\left(\frac{k^{2}}{a^{2}}+m_{\chi}^{2}+\frac{m_{I}^{2}}{M_{p}} \Phi(t) \sin \left(m_{\phi} t\right)\right) \chi_{k} \\
& =0,
\end{aligned}
$$

where $\Phi(t)$ is the amplitude of $\phi$. The amplitude $\Phi$ decreases as a consequence of the expansion of space. In the above, $k$ denotes the comoving wavenumber and $a(t)$ is the cosmological scale factor.

In a first step, we will put the above equation into the form of the well-known Mathieu equation. To absorb the expansion of space, we define a rescaled field via

$$
\eta_{k}=a^{3 / 2} \chi_{k} .
$$

Then, the equation of motion for $\eta_{k}$ becomes:

$\ddot{\eta}_{k}+\left(\frac{k^{2}}{a^{2}}+m_{\chi}^{2}+\frac{m_{I}^{2}}{M_{p}} \Phi(t) \sin \left(m_{\phi} t\right)-\Delta\right) \eta_{k}=0$,

where

$$
\Delta=\frac{3}{4} H^{2}+\frac{3}{2} \frac{\ddot{a}}{a}=\frac{3}{2}(1 / 2+(p-1) / p) H^{2},
$$

where for the second equality we have assumed that $a(t) \propto t^{p}$.

It is convenient to introduce a dimensionless time variable via

$$
z=\frac{1}{2} m_{\phi} t+\frac{\pi}{4}
$$

The differentiation with respect to $z$ will be denoted by a prime. In this case, the above equation (10) takes the form

$$
\eta_{k}^{\prime \prime}+\left(A_{k}-2 q \cos 2 z\right) \eta_{k}=0,
$$


where

$$
\begin{aligned}
A_{k} & =4 \frac{k^{2}}{m_{\phi}^{2} a^{2}}+4 \frac{m_{\chi}^{2}}{m_{\phi}^{2}}-4 m_{\phi}^{-2} \Delta \\
q & =2 \frac{m_{I}^{2} \Phi}{m_{\phi}^{2} M_{p}} .
\end{aligned}
$$

If it is justified to neglect the expansion of space compared to the rate of the processes which will be discussed in the following, then $\Delta$ vanishes, and the equation (13) takes the form of the Mathieu equation. Note that $k / a$ is the physical wavenumber.

\section{PARAMETRIC RESONANCE INSTABILITY}

The modulus field $\phi$ is frozen until the Hubble parameter $H$ drops to a value comparable to $m_{\phi}$. Then, $\phi$ begins to oscillate about $\phi=0$ with a frequency $m_{\phi}$, its amplitude $\Phi(t)$ being damped by the expansion of the spatial background and by the energy loss of $\phi$ to other fields. The second effect is a back-reaction effect which we will neglect. The condition required that the oscillation of $\phi$ begins before the time of nucleosynthesis is that the Hubble damping term in the equation of motion for $\phi$ becomes smaller than the force term $V^{\prime}(\phi)$ driving the oscillations. It yields

$$
H\left(T_{N S}\right)<m_{\phi},
$$

(where $T_{N S}$ is the temperature at which nucleosynthesis takes place) a condition which is satisfied for all masses in the dangerous range. Once the modulus field starts to oscillate, resonant excitation of all fields coupled to $\phi$ is possible, in particular the excitation of $\eta$.

The first instability we will study is the parametric resonance instability, first applied to the decay of the inflaton field in [23]. There are two types of resonance [24], broad parametric resonance and narrow parametric resonance. The condition for broad resonance is $q>1$. where $q$ is the parameter appearing in equation (13). This condition is satisfied for large values of the amplitude $\Phi$, namely for

$$
\Phi>\Phi_{b} \equiv M_{p}\left(\frac{m_{\phi}}{m_{I}}\right)^{2} .
$$

Evaluating this condition at the time when perturbative decay of the modulus field sets in, i.e. when $\Gamma=H$, and using for formula for $\Gamma$ applicable to our toy model (given below in (29)), we find that unless

$$
m_{\phi} \ll m_{I}\left(\frac{m_{I}}{10 M_{p}}\right)^{1 / 2},
$$

broad parametric resonance can relax but not solve the moduli problem without an additional decay channel being present. Both narrow resonance (discussed below) and the tachyonic decay discussed in the following section can provide the additional channel.

For smaller values of $\Phi$, we are in the domain of narrow parametric resonance. In this phase, the growth of $\eta_{k}$ is known $[29,30]$ :

$$
\eta_{k} \sim e^{q z} \sim e^{q m_{\phi} t / 2} .
$$

Only modes in narrow resonance bands are amplified, and the first such band is centered at a value of $k=k_{m}$ given by [29, 30]

$$
A_{k}=1, \quad k_{m}=\frac{m_{\phi}}{2}\left(1-\frac{4 m_{\chi}^{2}}{m_{\phi}^{2}}\right)^{1 / 2}
$$

from which it follows that the band does not exist unless $m_{\chi}<m_{\phi} / 2$. Other resonance bands occur for larger values of $A_{k}$ but are of higher order in perturbation theory and hence have a negligible effect.

The first condition for resonance to be effective is that the typical time scale for the growth of $\eta_{k}$ is shorter than the Hubble time, i.e.

$$
q m_{\phi}>H .
$$

In addition, one should take into account the change of the momentum as a result of the background expansion [26]. In a time interval $\delta t$, assuming $m_{\chi}<m_{\phi} / 4$, the change in the physical momentum $p=k / a(t)$ corresponding to the middle of the lowest resonance band is

$$
\Delta p=p H \Delta t \simeq \frac{m_{\phi}}{2} H \Delta t .
$$

The width of the resonance band is

$$
\Delta p=\frac{q m_{\phi}}{2} .
$$


Thus, $p$ remains in the resonance band during a time interval

$$
\Delta t \simeq q H^{-1} .
$$

To justify neglecting the expansion of space, we must require that the exponent in the growth factor (19) is at least 1 during this time interval. This leads to a more severe constraint on $q$ :

$$
q^{2} m_{\phi}>H \text {. }
$$

Inserting the value of $q$ from (15), we find that narrow resonance is efficient provided

$$
\Phi(t)>\Phi_{c}(t) \equiv \frac{\sqrt{H} M_{p} m_{\phi}^{3 / 2}}{m_{I}^{2}} .
$$

Since $\sqrt{H}$ decreases as $t^{-1 / 2}$ whereas in a radiation-dominated phase $\Phi(t)$ decreases only as $t^{-3 / 4}$, the narrow resonance decay channel eventually shuts off. In order that the moduli field does not dominate the energy density at the time of the shutoff at temperature $T$, the following condition needs to be satisfied:

$$
m_{\phi}^{2} \Phi_{c}^{2} \ll T^{4},
$$

which, inserting the expression (27) for $\Phi_{c}$, turns into the condition

$$
m_{\phi} \ll m_{I}\left(\frac{T^{2}}{m_{I} M_{p}}\right)^{1 / 5} .
$$

For narrow parametric resonance to solve the cosmological moduli problem, one needs to check that at the time of moduli decay (which occurs when $\Gamma \sim H$ ), the condition (28) still holds. The decay rate of $\phi$ for our Lagrangian (7) is given by

$$
\Gamma=\frac{m_{I}^{4}}{32 \pi M_{p}^{2} m_{\phi}} .
$$

Thus, the solution of the cosmological moduli problem requires:

$$
m_{\phi} \ll m_{I}\left(\frac{m_{I}}{10 M_{p}}\right)^{1 / 3} .
$$

Inserting the temperature of nucleosynthesis to get lower bound from (29) on the potentially dengerous mass range, and using the value $m_{I}=10^{2}$
$\mathrm{GeV}$, the problem is solved for values of $m_{\phi}$ satisfying

$$
10^{-7} \mathrm{GeV}<m_{\phi}<10^{-4} \mathrm{GeV} .
$$

In the context of our model (for $m_{I}=10^{2} \mathrm{GeV}$ ), moduli with masses smaller than $10^{-7} \mathrm{GeV}$ decay before time of nucleosynthesis and thus do not cause the problem. Note that this scaling of the decay rate with the mass of the decaying particle is going against the intuition that lighter moduli should decay later than heavier one. This curious aspect of our toy model decreases the potentially dangerous mass range, and this realization might be useful in some concrete models suffering from a moduli problem.

It appears at this point of our study that the period of narrow parametric resonance has the potential of solving the moduli problem for values of $m_{\phi}$ and $m_{I}$ which satisfy the relation given by (30). One issue which we have not taken into account is the fact that late moduli decay may provide a large source of non-thermal photons which could distort the black-body nature of the CMB. The constraints resulting from this effect must be studied in any concrete model with late-decaying moduli fields.

The previous analysis has missed a second important condition for the efficiency of narrow parametric resonance. It is not sufficient that the modes $\eta_{k}$ increase with a rate faster than $H$. Since the resonance occurs only in narrow bands $[29,30]$, it is important to check that the rate of energy increase integrated over all modes of $\eta$ be larger than the decrease in the energy density of $\phi$ taking into account the expansion of space alone. Otherwise, the energy stored in the moduli field would still scale as matter in spite of the exponential increase in the occupation number of certain field modes. This condition reads

$$
\dot{\rho_{\eta}}>H \rho_{\phi} .
$$

The rate of increase $\dot{\rho}_{\eta}$ in the energy density of $\eta$ can be estimated by considering the increase in the amplitude of all modes of $\eta$ in the lowest instability band. This band is located at $k \sim m_{\phi}$ and its width is given by $q m_{\phi}$. Since the rate of increase (from (19)) is $q m_{\phi}$ and since the initial mode (vacuum) energy is about $k$, we obtain

$$
\dot{\rho_{\eta}} \sim m_{\phi}^{5} q^{2} .
$$


Hence, the condition (32) for efficiency of the resonance process becomes

$$
m_{\phi}<m_{I} \frac{m_{I}^{2}}{T^{2}} \frac{m_{I}}{M_{p}}
$$

which is to be evaluated at the temperatures when the presence of moduli fields are dangerous for cosmology. Using, as before, the value $m_{I}=10^{2} \mathrm{GeV}$, and evaluating the above condition at the temperature of nucleosynthesis, we find that the condition is satisfied as long as the mass $m_{\phi}$ is smaller than about $10^{-5} \mathrm{GeV}$. The condition (34) becomes increasingly well satisfied at lower temperatures, and is no longer a concern at the time of recombination. Note that the condition (32) for the efficiency of narrow resonance is a very conservative one. As long as the first condition (25) is satisfied, the mode amplitude will grow, and hence the vacuum mode amplitude used in the above estimate should be replaced by the excited amplitude, thus relaxing the constraint by an exponential factor.

The problem, however, is that the condition (34) conflicts up to coefficients of order unity with the condition that the perturbative decay rate is negligible. Thus, in our toy model, and using the very conservative form of our conditions for the effectiveness of the resonance process, the narrow resonance decay channel is only effective near the time when the perturbative decay is also become important. This result, however, is a consequence of the particular scaling of $\Gamma$ with $m_{\phi}, \Gamma \propto m_{\phi}^{-1}$. In models where moduli decay to fermions, $\Gamma \propto m_{\phi}$, and, therefore, we expect those models do not suffer from this specific problem.

The above discussions neglected the expansion of the universe. Taking into account this expansion changes the Mathieu equation into a more general equation of Floquet type, and leads to a stochastic nature of the resonance process [26]. However, the property that the number of particles is growing exponentially at a rate given by (19) is preserved.

\section{TACHYONIC DECAY OF THE OSCILLATING MODULUS FIELD}

In the case of the decay of the inflaton field at the end of the period of inflation, it is known [27] that for certain models there is a tachyonic instability channel which is more efficient than para- metric resonance. In this section, we will study a similar process for moduli decay.

Let us return to the basic equation (13), with the values of the parameters $A_{k}$ and $q$ given by (14) and (15), respectively. We immediately see that for large values of $\Phi$, the effective $m^{2}$ term in the equation will be negative for part of the oscillation period of $\phi$. This tachyonic instability occurs provided

$$
\frac{m_{I}^{2} \Phi}{m_{\phi}^{2} M_{p}}>\frac{m_{\chi}^{2}}{m_{\phi}^{2}}
$$

The minimal value for which the tachyonic decay channel is open is given by setting the two sides in (35) equal and will be denoted by $\Phi_{m}$.

The condition under which the tachyonic decay channel can solve the moduli problem is then given by the condition

$$
m_{\phi}^{2} \Phi_{m}^{2} \ll T^{4},
$$

where $T$ is the temperature corresponding to the period one is interesting in. Evaluating (36) at the time of nucleosynthesis, we obtain

$$
m_{\phi} \ll T_{N S} \frac{T_{N S}}{M_{p}} \frac{m_{I}^{2}}{m_{\chi}^{2}} .
$$

The upper mass bound on $m_{\phi}$ for which the above tachyonic decay is effective thus depends sensitively on the ratio of $m_{I}$ and $m_{\chi}$. Unless the latter mass is much smaller than the former, the tachyonic decay channel cannot reduce the amplitude of moduli oscillations to a level consistent with the observational constraints.

The range of values of $m_{\phi}$ for which the two decay channels - narrow parametric resonance (neglecting for a moment the issue that in our model it starts to be efficient together with the perturbative decay) and tachyonic decay - are open depends on the values of the masses. While if $m_{\chi}>m_{\phi}$, the only allowed channel is the tachyonic one, the narrow parametric resonance works for a wider range of masses $m_{\phi}$ when $m_{\chi} \sim m_{\phi}$. The later can be seen by inserting $m_{\chi}=m_{\phi}$ into (37) and comparing with (28). However, as the value of $m_{\chi}$ is reduced, the range of masses for which the tachyonic decay channel is open grows and begins to dominate over that of narrow parametric resonance. Tachyonic decay can be made to work even for $m_{I} \sim m_{\phi}$. 
For values of $m_{\phi}$ for which both decay channels are open, the tachyonic decay is more efficient for two reasons. First, it leads to the excitation of all long wavelength modes, and not just to modes in a narrow resonance band. Modes with

$$
k_{p}^{2}<m_{I}^{2} \frac{\Phi}{M_{p}} \equiv k_{c r i t}^{2}
$$

are excited. For such modes, the value of $\eta_{k}$ increases with a maximal rate given by

$$
\eta_{k} \sim \exp (\sqrt{q} z),
$$

which for $q<1$ is a larger rate than that which occurs for modes in the resonance band during narrow resonance (see (19)). This is the second reason for the larger efficiency of the tachyonic decay channel.

Let us estimate the energy density $\rho_{\eta}$ stored in the quanta produced during the tachyonic decay process. The phase space of modes which are excited tachyonically is of the order $k_{\text {crit }}^{3}$. Each mode grows with a rate which varies in time, the maximal rate being given by (39), and the growth occurs for approximately half the oscillation period (the period during which the effective square mass is negative). The mean growth rate is given by $1 / \sqrt{2}$ of the maximal rate. Thus

$$
\rho_{\eta}(t) \sim m_{I}^{4} \frac{\Phi^{2}}{M_{p}^{2}} \exp \left(\frac{1}{\sqrt{8}} m_{I} \sqrt{\Phi / M_{P}} t\right) .
$$

The prefactor in front of the exponential factor is much larger than the corresponding factor in the case of the narrow resonance decay process.

\section{DISCUSSION AND CONCLUSIONS}

In this paper we have studied two nonperturbative decay processes which can substantially dilute the density of dangerous moduli fields. Both processes occur during the phase in which the moduli fields oscillate about their ground states. The first non-perturbative process is parametric resonant excitation of fields coupled to the modulus field. In this paper we modeled such fields as a scalar field $\chi$ coupled to the modulus field $\phi$ via dimension five operators suppressed by the Planck mass. The second decay process is tachyonic decay which makes use of the fact that the effective square mass of $\chi$ in the presence of the oscillating $\phi$ field is negative for part of the oscillation period. These decay processes are analogous to the parametric resonant decay of the inflaton during reheating [23], and to tachyonic preheating [27], respectively.

We have established the conditions under which either of the two decay processes can solve the moduli problem, i.e. reduce the energy density of the modulus field to values consistent with big bang nucleosynthesis. It appears that narrow parametric resonance has the potential to solve the modulus problem but, for the toy model considered here, and using very conservative conditions for the effectiveness of the resonance process, it happens only at the time when the pertubative decay rate also becomes important. Undoubtedly, tachyonic decay successfully solves the problem given masses of decay products which are much smaller than the scale of interaction $-m_{I}$. Moreover, the tachyonic decay channel allows for excitation of particles with masses heavier than that of the decaying particle. Depending on the values of the other masses in the Lagrangian, either of the two decay processes can be open for a wider range of masses $m_{\phi}$. For values of $m_{\phi}$ for which both decay channels are open, the tachyonic decay is much more efficient, as is true in the case of the decay of the inflaton. Note that the presence of an interaction term in the Lagrangian linear in $\phi$ was important in order to obtain the tachyonic decay.

It will be of great interest to study the applicability of these decay channels to concrete models with moduli fields. This work is left for future research.

The form of the potential in our toy model Lagrangian inevitably suggests inflation at low scales. This natural source of inflation does not only dilute heavier relics but could also mitigate the flatness, horizon and entropy problems. It needs to be studied whether this type of models can provide a successful reheating mechanism, if the inflaton has only gravitationally suppressed interactions. Once again, non-perturbative instabilities like those used in preheating $[23,24,25,26,27]$ are likely to be successful. If the modulus field comes to dominate the energy density of the universe for some period (without necessarily leading to inflation), it can provide a candidate for the curvaton (see e.g. [31] for an extensive discussion of moduli fields as 
candidates for the curvaton).

\section{Acknowledgments}

This work is supported by NSERC. The research of N.S. is also supported in part by McGill Univer- sity under a McGill Graduate Student Fellowship. We would like to thank the Perimeter Institute (N.S.) and the Yukawa Institute for Theoretical Physics at the University of Kyoto (and in particular Misao Sasaki) (R.B.) for kind hospitality during the time when this project was completed.
[1] G. D. Coughlan, W. Fischler, E. W. Kolb, S. Raby and G. G. Ross, Phys. Lett. B 131, 59 (1983).

[2] J. R. Ellis, D. V. Nanopoulos and M. Quiros, Phys. Lett. B 174, 176 (1986).

[3] B. de Carlos, J. A. Casas, F. Quevedo and E. Roulet, Phys. Lett. B 318, 447 (1993) [arXiv:hep-ph/9308325].

[4] T. Banks, D. B. Kaplan and A. E. Nelson, Phys. Rev. D 49, 779 (1994) [arXiv:hep-ph/9308292].

[5] A. S. Goncharov, A. D. Linde and M. I. Vysotsky, Phys. Lett. B 147, 279 (1984).

[6] A. D. Linde, Phys. Lett. B 116, 335 (1982).

[7] A. Vilenkin and L. H. Ford, Phys. Rev. D 26, 1231 (1982).

[8] G. F. Giudice, A. Riotto and I. I. Tkachev, JHEP 0106, 020 (2001) [arXiv:hep-ph/0103248].

[9] G. F. Giudice, I. Tkachev and A. Riotto, JHEP 9908, 009 (1999) [arXiv:hep-ph/9907510].

[10] G. N. Felder, L. Kofman and A. D. Linde, JHEP 0002, 027 (2000) [arXiv:hep-ph/9909508].

[11] J. R. Ellis, A. D. Linde and D. V. Nanopoulos, Phys. Lett. B 118, 59 (1982).

[12] M. Dine, L. Randall and S. Thomas, Phys. Rev. Lett. 75, 398 (1995) [arXiv:hep-ph/9503303].

[13] L. Randall and S. Thomas, Nucl. Phys. B 449, 229 (1995) [arXiv:hep-ph/9407248].

[14] D. H. Lyth and E. D. Stewart, Phys. Rev. D 53, 1784 (1996) [arXiv:hep-ph/9510204].

[15] T. Asaka and M. Kawasaki, Phys. Rev. D 60, 123509 (1999) [arXiv:hep-ph/9905467].

[16] T. Asaka, M. Kawasaki and M. Yamaguchi, Phys. Lett. B 451, 317 (1999) [arXiv:hep-ph/9810334].

[17] M. Kawasaki and F. Takahashi, Phys. Lett. B 618, 1 (2005) [arXiv:hep-ph/0410158].

[18] M. Dine, W. Fischler and D. Nemeschansky, Phys.
Lett. B 136, 169 (1984).

[19] G. D. Coughlan, R. Holman, P. Ramond and G. G. Ross, Phys. Lett. B 140, 44 (1984).

[20] A. D. Linde, Phys. Rev. D 53, 4129 (1996) [arXiv:hep-th/9601083].

[21] L. Kofman, A. Linde, X. Liu, A. Maloney, L. McAllister and E. Silverstein, JHEP 0405, 030 (2004) [arXiv:hep-th/0403001].

[22] S. Watson, Phys. Rev. D 70, 066005 (2004) [arXiv:hep-th/0404177].

[23] J. H. Traschen and R. H. Brandenberger, Phys. Rev. D 42, 2491 (1990).

[24] L. Kofman, A. D. Linde and A. A. Starobinsky, Phys. Rev. Lett. 73, 3195 (1994) [arXiv:hepth/9405187].

[25] Y. Shtanov, J. H. Traschen and R. H. Brandenberger, Phys. Rev. D 51, 5438 (1995) [arXiv:hep$\mathrm{ph} / 9407247]$.

[26] L. Kofman, A. D. Linde and A. A. Starobinsky, Phys. Rev. D 56, 3258 (1997) [arXiv:hepph/9704452].

[27] G. N. Felder, J. Garcia-Bellido, P. B. Greene, L. Kofman, A. D. Linde and I. Tkachev, Phys. Rev. Lett. 87, 011601 (2001) [arXiv:hepph/0012142].

[28] P. B. Greene and L. Kofman, Phys. Lett. B 448 , 6 (1999) [arXiv:hep-ph/9807339].

[29] L. Landau and I. Lifshitz, Mechanics (Pergamon, Oxford, 1960).

[30] V. Arnold, Mathematical Methods of Classical Mechanics (Springer, New York, 1974).

[31] K. Enqvist and A. Mazumdar, Phys. Rept. 380, 99 (2003) [arXiv:hep-ph/0209244]. 